\title{
Water flow on vegetated hill. 1D shallow water equation type model
}

\author{
Stelian Ion, Dorin Marinescu, Anca Veronica Ion, \\ Stefan Gicu Cruceanu, Virgil Iordache
}

\begin{abstract}
A mathematical model for the water flow on a hill covered by variable distributed vegetation is proposed in this article. The model takes into account the variation of the geometrical properties of the terrain surface, but it assumes that the surface exhibits large curvature radius. After describing some theoretical properties for this model, we introduce a simplified model and a well-balanced numerical approximation scheme for it. Some mathematical properties with physical relevance are discussed and finally, some numerical results are presented.
\end{abstract}

\section{Introduction}

The water accumulation on the surface of the soil is a complex process involving, among others, rain and infiltration. Taking into consideration only these two phenomena, it is obvious that a water layer is produced by the rain drops if the rain rate exceeds the infiltration rate, and this stratum of exceeding water moves down the hill on the soil surface. Such flow (usually named hortonian flow) was modelled in [7].

The presence of plants on a hill creates a resistance force to the water flow and influences the process of water accumulation on the soil surface. The

Key Words: Hyperbolic system, finite volume method, well-balanced scheme, energy dissipation.

2010 Mathematics Subject Classification: Primary 35Q35, 76S99; Secondary 34A34, 35L40, 65M08.

Received: November, 2014.

Revised: February, 2015.

Accepted: February, 2015. 
large diversity of growing plants on a hill makes the elaboration of an unitary model for the water flow over the soil covered by plants very difficult. In the analyzed model of this paper, we assume that the plants form a dense net of rigid vertical tubes and that the water fills the "voided" space up to a level not higher than these plant tubes (see Figure 1 for some graphical details).

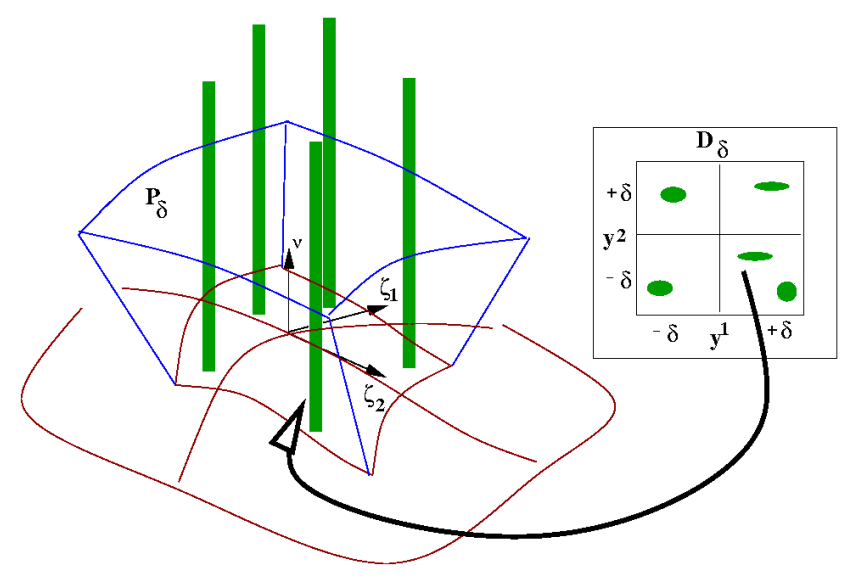

Figure 1: The representative element of the volume $P_{\delta}$ used for averaging. The bottom surface $z\left(y^{1}, y^{2}\right)$ of $P_{\delta}$ has a representative width $\delta$ along two orthogonal directions on this surface. $\xi_{1}, \xi_{2}$ are the tangent vectors at $z\left(y^{1}, y^{2}\right)$ and $\nu$ is the unit normal to this surface. The water depth $h$ associated to $P_{\delta}$ is the averaged value of the physical water depth $\widetilde{h}$ inside $P_{\delta}$.

One assumes that the terrain exhibits topographic variation but with small curvature, and that the terrain surface is locally almost plane. One also supposes that the viscosity of fluid and the fluctuation of the velocity field have a small effect as compared to the bed friction and plant resistance. In what follows, we will write the model equations (1) in a soil surface based curvilinear co-ordinates system with $\left(y^{1}, y^{2}\right)$ the surface coordinates. The vector or tensor components relative to this co-ordinate system are indexed by $a, b, c, \ldots$; $\beta_{c a}$ stands for the covariant components of the metric tensor, $\gamma_{a b}^{c}$ are the Christophel symbols, $\beta=\sqrt{\operatorname{det}(\beta . .)}$, and $\beta \mathrm{d} y^{1} \mathrm{~d} y^{2}$ is the area element of the soil surface. The contravariant components of the velocity are denoted by $v^{a}$, the covariant components of it by $v_{a}$, and they are related by $v_{a}=\beta_{a c} v^{c}$. Starting from the general principles for mass and momentum balance laws and invoking the above assumptions, one obtains the following basic model 
for water flow on vegetated hills

$$
\begin{aligned}
\partial_{t}(h \beta \theta)+\partial_{a}\left(h \beta \theta v^{a}\right) & =\beta\left(\mathfrak{m}_{r}-\theta \mathfrak{m}_{i}\right), \\
\partial_{t}\left(h \theta \beta v_{c}\right)+\partial_{a}(\theta & \left.\beta h v_{c} v^{a}\right)+ \\
+h \beta \theta \partial_{c} w & =h \theta \beta \gamma_{a c}^{b} v^{a} v_{b}-\beta \mathcal{K}(h, \theta)|v| v_{c}, \quad c=1,2,
\end{aligned}
$$

where $\theta$ is the porosity of the vegetation cover; $w$ is, roughly speaking, the altitude of the water surface; $\mathfrak{m}_{r}, \mathfrak{m}_{i}$ are the water supply rate (from rain) and the water loss rate (by infiltration), respectively.

These equations were obtained by averaging the macroscopic variables over a representative element of volume $P_{\delta}$ placed on the land surface $z\left(y^{1}, y^{2}\right)$ of the flow and illustrated in Figure 1. The reader is referred to [7] for details concerning the deduction of $(1)$.

The parameter function $\mathcal{K}(h, \theta)$ is given by

$$
\mathcal{K}(h, \theta)=\alpha_{p} h(1-\theta)+\alpha_{s} \theta
$$

where $\alpha_{p}$ and $\alpha_{s}$ quantifies the water-plants and water-soil interactions, respectively. The model described by equations (1) is mathematically too complicated for many practical applications, but it is a good basic model to generate simplified models of certain realistic problems. Such models can be obtained by considering stronger assumptions on the soil surface topography and the structure of the plant cover. The porous analogy for the fluid-plant physical system was also used in $[1,6,8,9,11,15]$ especially for the case of submerged vegetation where the flow is assumed to be plan parallel. For general topography, a mathematical model was obtained in [2]. In what follows, we introduce a different variant (still simplified) of the full model which now allows small variations in soil topography and plant porosity.

\section{The simplified model}

Assume that the soil surface is reprezented by

$$
x^{1}=y^{1}, \quad x^{2}=y^{2}, \quad x^{3}=z\left(y^{1}, y^{2}\right),
$$

and the first derivatives of the function $z\left(y^{1}, y^{2}\right)$ are small quantities.

\section{Assumptions:}

(a) Geometrical assumptions: $|\nabla z|^{2} \approx 0, \nabla^{2} z \approx 0$.

(b) Vegetation structure: $\theta \approx \theta_{0}$. 
Under the above assumptions, equations (1) are approximated by

$$
\begin{aligned}
\partial_{t} h+\partial_{a}\left(h v^{a}\right) & =\mathfrak{M}, \\
\partial_{t}\left(h v_{a}\right)+\partial_{b}\left(h v_{a} v^{b}\right)+h \partial_{a} w & =-\mathcal{K}(h, \theta)|v| v_{a}, \quad a=\overline{1,2},
\end{aligned}
$$

where

$$
\mathcal{K}(h, \theta)=\alpha_{p} h\left(\frac{1}{\theta}-1\right)+\alpha_{s}, \quad \mathfrak{M}=\frac{\mathfrak{m}_{r}}{\theta}-\mathfrak{m}_{i}, \quad w=g\left[z\left(y^{1}, y^{2}\right)+h\right] .
$$

By using the "flatness" assumption (a), one can identify the covariant and contravariant components of the velocity, $v_{a}=\delta_{a c} v^{c}$. (3) is a hyperbolic system of equations with source term for which there is an energy function that is conserved in the absence of plants and water-soil friction. Moreover, the model preserves the steady state of a lake. These properties are more precisely formulated in what follows.

Proposition 1. The reduced model (3) of the water flow on vegetated hill is of hyperbolic type with source terms.

(a) Its conservative form is given by

$$
\partial_{t} \mathcal{H}(\boldsymbol{u})+\partial_{a} \mathcal{F}^{a}(\boldsymbol{u})=\mathcal{P}(y, \boldsymbol{u})
$$

where $\boldsymbol{u}=\left(h, v^{1}, v^{2}\right)^{\mathrm{T}}, \mathcal{H}=\left(h, h v^{1}, h v^{2}\right)^{\mathrm{T}}$ and

$\mathcal{F}=\left(\begin{array}{cc}h v^{1} & h v^{2} \\ h v_{1} v^{1}+g h^{2} / 2 & h v_{1} v^{2} \\ h v_{2} v^{1} & h v_{2} v^{2}+g h^{2} / 2\end{array}\right), \mathcal{P}=\left(\begin{array}{c}\mathfrak{M} \\ -h g \partial_{1} z-\mathcal{K}(h, \theta)|v| v_{1} . \\ -h g \partial_{2} z-\mathcal{K}(h, \theta)|v| v_{2} .\end{array}\right)$

(b) For any unitary vector $\boldsymbol{n} \in \mathbb{R}^{3}$, the eigenvalue problem

$$
\left(\frac{\partial}{\partial u^{i}} \mathcal{F}^{j a} n_{a}-\lambda \frac{\partial}{\partial u^{i}} \mathcal{H}^{j}\right) r^{i}=0, \quad i=\overline{1,3}
$$

has three distinct eginvalues:

$$
\lambda_{-}=v^{a} n_{a}-\sqrt{g h}, \quad \lambda_{0}=v^{a} n_{a}, \quad \lambda_{+}=v^{a} n_{a}+\sqrt{g h} .
$$

Proof. The conservative form (5) results from (3) by direct calculation. To prove the existence of the eigenvalues, one observes that

$$
\frac{\partial}{\partial u^{i}} \mathcal{F}^{j a} n_{a}-\lambda \frac{\partial}{\partial u^{i}} \mathcal{H}^{j}=\left(\begin{array}{ccc}
\psi & h n^{1} & h n^{2} \\
v_{1} \psi+g h n_{1} & h \psi+h v_{1} n^{1} & h v_{1} n^{2} \\
v_{2} \psi+g h n_{2} & h v_{2} n^{1} & h \psi+h v_{2} n^{2}
\end{array}\right),
$$

where $\psi=v^{a} n_{a}-\lambda$, and then by simple calculation one obtains (6). 
Proposition 2. The following properties hold for (3):

(a) in the absence of mass sources $(\mathfrak{M}=0)$, the system preserves the steady state of a lake

$$
\partial_{a}\left(x^{3}+h\right)=0, \quad v_{a}=0, \quad a=\overline{1,2} ;
$$

(b) there exists a conservative form for the equation of the energy dissipation

$$
\partial_{t}(h \mathcal{E})+\partial_{a}\left(h v^{a} \mathcal{E}+g \frac{h}{2}\right)=\mathfrak{M}\left(-\frac{1}{2}|v|^{2}+w\right)-\mathcal{K}|v|^{3},
$$

where

$$
\mathcal{E}:=\frac{1}{2}|v|^{2}+g\left(x^{3}+\frac{h}{2}\right) ;
$$

(c) Bernoulli law. In a steady state, in the absence of mass source and friction force, the total energy

$$
\mathcal{E}^{\mathrm{t}}=\frac{1}{2}|v|^{2}+g x^{3}+p(y, h)
$$

is constant along a current line

$$
v^{a} \partial_{a} \mathcal{E}^{\mathrm{t}}=0
$$

Proof. The assertion (a) results immediately since the steady state of a lake obviously verifies (3). For (b), we multiply the momentum balance equations (3b) by $v^{a}$, and then, using the mass balance equation (3a) one writes

$$
h\left(\partial_{t}\left(|v|^{2} / 2\right)+v^{a} \partial_{a}\left(|v|^{2} / 2+w\right)\right)=-(\mathfrak{M}+\mathcal{K}|v|)|v|^{2} .
$$

Now, using again the mass balance equation one obtains the equation (7) of the energy dissipation. Bernoulli law results from (7) and the fact that in the shallow water approximation model the pressure field increases linearly with the water depth, $p(y, h)=g h(y)$.

The presence of the plants and the existence of a frictional interaction between water and soil induce an energy loss. In order to highlight such phenomenon, let us consider a domain $\Omega$ and $\boldsymbol{n}$ the outward oriented normal unitary vector to the boundary $\partial \Omega$. Assume also that $\partial \Omega$ consists of an impermeable region $\Gamma_{1}$ and an exit region $\Gamma_{2}$, i.e.

$$
\partial \Omega=\Gamma_{1} \cup \Gamma_{2}, \quad \boldsymbol{n} \cdot \boldsymbol{v}=0 \text { on } \Gamma_{1}, \quad \text { and } \quad \boldsymbol{n} \cdot \boldsymbol{v}>0 \text { on } \Gamma_{2},
$$

where one of these regions can be the empty set. 
Proposition 3 (Energy dissipation). If there is no mass production, then the energy of $\Omega$ is a decreasing function with respect to time, i.e.

$$
\partial_{t} \int_{\Omega} h \mathcal{E} \mathrm{d} x<0 .
$$

Proof. The inequality (9) results immediately after integrating the equation (7) of the energy dissipation

$$
\partial_{t} \int_{\Omega} h \mathcal{E} \mathrm{d} x+\int_{\partial \Omega} h \boldsymbol{v} \cdot \boldsymbol{n} \mathcal{E}^{t} \mathrm{~d} s=-\int_{\Omega} \mathcal{K}|v|^{3} \mathrm{~d} x
$$

and observing that the second term of the left hand side is positive.

There is a large amount of literature devoted to hyperbolic systems with source term. We mention [2] and [13] as being closest to our problem and [10] for the general problem of the existence of weak solution in the case of non-conservative product.

\section{$3 \quad$ FVM approximation of 1D simplified model}

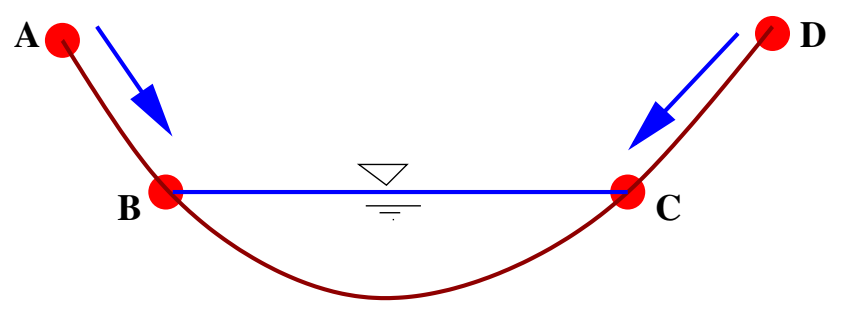

Figure 2: A possible (often encountered in the water flow in a hydrographic basin) physical configuration that implies singular point in the mathematical model. Water runs downslope and it accumulates in the deep valey. On the surface branches $A B \cup C D$ the water depth is zero and in the region corresponding to the segment $B C$ one has $u=0$ and $z+h=$ const.

For the most real cases, the soil surface exibits convex and concave regions. The presence of the concave ones generates lakes (e.g. as in Figure 2) which are local equilibrium points for system (3). To numerically capture the solution behavior in the neighborhood of such equilibrium points is a very challenging issue and will be the main subject of the next sections. 
In what follows, we propose a method which uses only one set of grid points for the discretization of both water depth and velocity functions. Let $x_{i}$ be the center of the volume element $V_{i}:=\left(x_{i-1 / 2}, x_{i+1 / 2}\right)$. We introduce the averaged quantities

$$
\begin{gathered}
h_{i}(t):=\frac{1}{\triangle x} \int_{x_{i-1 / 2}}^{x_{i+1 / 2}} h(t, x) \mathrm{d} x, \quad u_{i}(t):=\frac{1}{\triangle x} \int_{x_{i-1 / 2}}^{x_{i+1 / 2}} u(t, x) \mathrm{d} x, \\
h u_{i}:=\frac{1}{\triangle x} \int_{x_{i-1 / 2}}^{x_{i+1 / 2}} h u(t, x) \mathrm{d} x .
\end{gathered}
$$

The discrete ODE model is given by

$$
\begin{aligned}
\triangle x \partial_{t} h_{i} & =-\left[(h u)_{i+1 / 2}-(h u)_{i-1 / 2}\right]+\triangle x \mathfrak{M}_{i}, \\
\triangle x \partial_{t}\left(h u_{i}\right) & =-\left[\left(h u^{2}\right)_{i+1 / 2}-\left(h u^{2}\right)_{i-1 / 2}\right]-f_{i}-\triangle x \mathcal{K}_{i}\left|u_{i}\right| u_{i},
\end{aligned}
$$

where

$$
f_{i}=\int_{x_{i-1 / 2}}^{x_{i+1 / 2}} h \partial_{x} w \mathrm{~d} x
$$

Upwind approximation: The upwind scheme is defined by

$$
\triangle x \partial_{t} U_{i}=\mathcal{F}_{i+1 / 2}-\mathcal{F}_{i-1 / 2}+\frac{1}{2}\left(\mathcal{S}_{i+1 / 2}+\mathcal{S}_{i-1 / 2}\right)+\triangle x \mathcal{T}_{i}
$$

where

$$
\begin{gathered}
U_{i}=\left(\begin{array}{c}
h_{i} \\
h_{i} u_{i}
\end{array}\right), \quad \mathcal{F}_{i+1 / 2}:=\mathcal{F}\left(U_{i}, U_{i+1}\right)=-\left(\begin{array}{c}
h_{i+1 / 2} \bar{u}_{i+1 / 2} \\
h_{i+1 / 2} \bar{u}_{i+1 / 2}^{2}
\end{array}\right) \\
\mathcal{S}_{i+1 / 2}:=\mathcal{S}\left((h, w)_{i},(h, w)_{i+1}\right)=-\left(\begin{array}{c}
0 \\
h_{i+1 / 2}^{s}\left(w_{i+1}-w_{i}\right)
\end{array}\right) \\
\mathcal{T}_{i}:=\left(\begin{array}{c}
\mathfrak{M}_{i} \\
-\mathcal{K}_{i}\left|u_{i}\right| u_{i}
\end{array}\right),
\end{gathered}
$$

with

$$
\begin{aligned}
& \bar{u}_{i+1 / 2}=\frac{u_{i+1}+u_{i}}{2}, \quad h_{i+1 / 2}=\left\{\begin{array}{ll}
h_{i}, & \bar{u}_{i+1 / 2}>0 \\
h_{i+1}, & \bar{u}_{i+1 / 2}<0
\end{array},\right. \\
& h_{i+1 / 2}^{s}= \begin{cases}h_{i+1 / 2}, & \bar{u}_{i+1 / 2} \neq 0 \\
h_{i+1}, & \bar{u}_{i+1 / 2}=0, \\
h_{i}, & \bar{u}_{i+1 / 2}=0, w_{i}>w_{i+1}\end{cases}
\end{aligned}
$$


Proposition 4. The upwind scheme (11)-(15) is consistent and its approximation order is $\mathcal{O}(\triangle x)$. In addition, if the mass sources are missing, then the positive cone $h_{i} \geq 0$ is a time invariant set.

Proof. To prove the consistence of the scheme, it is sufficient to show that

(i) $\mathcal{F}(U, U)=\left(\begin{array}{c}h u \\ h u^{2}\end{array}\right)$;

(ii) if $\left\{\begin{array}{ll}h_{i-1}=h-\triangle x \partial_{x} h+\mathcal{O}\left(\triangle^{2} x\right) & \\ h_{i+1}=h+\triangle x \partial_{x} h+\mathcal{O}\left(\triangle^{2} x\right) \\ w_{i-1}=w-\triangle x \partial_{x} w+\mathcal{O}\left(\triangle^{2} x\right) \\ w_{i+1}=w+\triangle x \partial_{x} w+\mathcal{O}\left(\triangle^{2} x\right)\end{array}\right.$, then $\left.\quad \begin{array}{c}\frac{1}{2}\left(\mathcal{S}_{i+1 / 2}+\mathcal{S}_{i-1 / 2}\right)= \\ 0 \\ h \partial_{x} w \triangle x+\mathcal{O}\left(\triangle^{2} x\right)\end{array}\right)$

Note that the terms from the r.h.s. of the equalities from (ii) are calculated in $x=x_{i}$. (i) and (ii) can be easily verified by straightforward calculations.

Also, in the absence of a mass source, one can write

$$
\triangle x \partial_{t} h_{i}=-\left(h_{i+1 / 2} \bar{u}_{i+1 / 2}-h_{i-1 / 2} \bar{u}_{i-1 / 2}\right)
$$

and observe that if $h_{i}$ equals zero then $\partial_{t} h_{i} \geq 0$.

One type of equilibrium point for our equations consists of a sequence of local lakes and dry regions. For simplicity, we consider an equilibrium point consisting of a single lake flanked by dry regions.

Proposition 5 (Steady lake). Let $N$ be the number of volume elements. Assume that the mass source $\mathcal{M}_{i}$ is missing and the discrete data $\mathcal{P}=\left\{\left(h_{i}, u_{i}\right)\right\}_{i=1, N}$ are such that there exists a set of consecutive indexes $\mathcal{J}=\left\{i_{0}, i_{0}+1, \ldots, i_{0}+l\right\}$, such that

(a) $z_{i}+h_{i}=\alpha, \forall i \in \mathcal{J}$,

(b) $z_{i} \geq \alpha, h_{i}=0, \forall i \notin \mathcal{J}$,

(c) $u_{i}=0, i=\overline{1, N}$.

Then $\mathcal{P}$ is an equilibrium point of ODEs (11).

Proof. The proposition is proved if one shows that the r.h.s. of (11) is zero for the equilibrium point $\mathcal{P}$. It is clear that $\mathcal{F}_{i+1 / 2}-\mathcal{F}_{i-1 / 2}=0$. Also, if $i \notin\left\{i_{0}-1, i_{0}, i_{0}+l, i_{0}+l+1\right\}$, then $\mathcal{S}_{i+1 / 2}+\mathcal{S}_{i-1 / 2}=0$ because all $h^{s}$ vanish inside the dry regions, while $w$-differences vanish inside the lake domain. At the boundary of the lake, i.e. for $i \in\left\{i_{0}-1, i_{0}, i_{0}+l, i_{0}+l+1\right\}$, we have $\mathcal{S}_{i+1 / 2}=0=\mathcal{S}_{i-1 / 2}$ since, for both of them, at least one of their factors (either $h^{s}$ or $w$-difference) vanishes. 
Proposition 6 (Energy dissipation). Let $N$ be the number of volume elements. Consider the boundary conditions given by

$$
u_{1}=0=u_{N}
$$

Let us introduce the independent variables $h_{i}, m_{i}$, with $m_{i}:=h_{i} u_{i}$ and define the discrete energy functions

$$
\mathcal{E}_{i}\left(h_{i}, m_{i}\right)=\frac{1}{2} \frac{m_{i}^{2}}{h_{i}}+\frac{1}{2} g h_{i}^{2}+g x_{i}^{3} h_{i}, \quad \mathcal{E}=\sum_{i=1}^{N} \triangle x \varepsilon_{i} .
$$

Let $\left\{\left(h_{i}^{0}, u_{i}^{0}\right)\right\}_{i=\overline{1, N}}$ be the initial data for system (11) and $\mathcal{E}$ the energy function corresponding to the solution of (11) with boundary conditions (16). Then $\mathcal{E}$ is a decreasing function with respect to the time variable, i.e.

$$
\frac{\mathrm{d}}{\mathrm{d} t} \mathcal{E}<0
$$

Proof. One has

$$
\frac{\mathrm{d}}{\mathrm{d} t} \mathcal{E}=\sum_{i} \triangle x \frac{\mathrm{d}}{\mathrm{d} t} \mathcal{E}_{i}=\sum_{i} \triangle x\left(\frac{\partial \mathcal{E}_{i}}{\partial h_{i}} \frac{\mathrm{d} h_{i}}{\mathrm{~d} t}+\frac{\partial \mathcal{E}_{i}}{\partial m_{i}} \frac{\mathrm{d} m_{i}}{\mathrm{~d} t}\right)
$$

where

$$
\frac{\partial \mathcal{E}_{i}}{\partial h_{i}}=w_{i}-\frac{1}{2} u_{i}^{2}, \quad \frac{\partial \mathcal{E}_{i}}{\partial m_{i}}=u_{i}
$$

and therefore

$$
\begin{aligned}
\triangle x\left(\frac{\partial \mathcal{E}_{i}}{\partial h_{i}} \frac{\mathrm{d} h_{i}}{\mathrm{~d} t}+\frac{\partial \mathcal{E}_{i}}{\partial m_{i}} \frac{\mathrm{d} m_{i}}{\mathrm{~d} t}\right) & =\left(w_{i}-\frac{1}{2} u_{i}^{2}\right) \triangle x \frac{\mathrm{d} h_{i}}{\mathrm{~d} t}+u_{i} \triangle x \frac{\mathrm{d} m_{i}}{\mathrm{~d} t}= \\
& =S_{i}+H_{i}-\triangle x \mathcal{K}\left|u_{i}\right|^{3}
\end{aligned}
$$

where

$$
\begin{array}{r}
S_{i}:=-w_{i}\left(h_{i+1 / 2} \bar{u}_{i+1 / 2}-h_{i-1 / 2} \bar{u}_{i-1 / 2}\right)-u_{i} \frac{1}{2}\left[h_{i+1 / 2}^{s}\left(w_{i+1}-w_{i}\right)+\right. \\
\left.+h_{i-1 / 2}^{s}\left(w_{i}-w_{i-1}\right)\right]
\end{array}
$$

and

$$
\begin{array}{r}
H_{i}:=\frac{1}{2} u_{i}^{2}\left(h_{i+1 / 2} \bar{u}_{i+1 / 2}-h_{i-1 / 2} \bar{u}_{i-1 / 2}\right)-u_{i}\left[h_{i+1 / 2}\left(\bar{u}_{i+1 / 2}\right)^{2}-\right. \\
\left.-h_{i-1 / 2}\left(\bar{u}_{i-1 / 2}\right)^{2}\right] .
\end{array}
$$


With the above notations, we can now write

$$
\frac{\mathrm{d}}{\mathrm{d} t} \mathcal{E}=\sum_{i} S_{i}+\sum_{i} H_{i}-\sum_{i} \triangle x \mathcal{K}\left|u_{i}\right|^{3} .
$$

Using the boundary conditions (16) and straightforward calculations, we get

$$
\begin{aligned}
\sum_{i} S_{i}= & \sum_{i=1}^{N-1} h_{i+1 / 2} \bar{u}_{i+1 / 2}\left(w_{i+1}-w_{i}\right)- \\
& -\sum_{i=1}^{N-1} h_{i+1 / 2}^{s} \bar{u}_{i+1 / 2}\left(w_{i+1}-w_{i}\right)= \\
= & \sum_{i=1}^{N-1}\left(h_{i+1 / 2}-h_{i+1 / 2}^{s}\right) \bar{u}_{i+1 / 2}\left(w_{i+1}-w_{i}\right)
\end{aligned}
$$

and with the definition (15) of $h^{s}$ one can easily conclude that

$$
\sum_{i} S_{i}=0
$$

Also

$$
\begin{aligned}
\sum_{i} H_{i}= & -\frac{1}{2} \sum_{i=1}^{N-1} h_{i+1 / 2} \bar{u}_{i+1 / 2}\left(u_{i+1}^{2}-u_{i}^{2}\right)+ \\
& +\sum_{i=1}^{N-1} h_{i+1 / 2}\left(\bar{u}_{i+1 / 2}\right)^{2}\left(u_{i+1}-u_{i}\right)
\end{aligned}
$$

and using again (15), we obtain

$$
\sum_{i} H_{i}=0
$$

Now (18), (19) and (20) give

$$
\frac{\mathrm{d}}{\mathrm{d} t} \mathcal{E}=-\sum_{i} \triangle x \mathcal{K}\left|u_{i}\right|^{3}
$$

which proves the proposition.

Remark 1. Unfortunately, the energy disipates at a very slow rate when the trajectory of ODEs approaches the equilibrium state, and therefore, from a numerical point of view, one faces with small oscillations of the free surface of the lake. 


\section{Artificial viscosity}

In order to eliminate these oscillations, we introduce artificial viscosity in (11) and obtain the following system of ODEs

$$
\triangle x \partial_{t} U_{i}=\mathcal{F}_{i+1 / 2}^{v}-\mathcal{F}_{i-1 / 2}^{v}+\frac{1}{2}\left(\mathcal{S}_{i+1 / 2}+\mathcal{S}_{i-1 / 2}\right)+\triangle x \mathcal{T}_{i},
$$

where

$$
\mathcal{F}^{v}\left(U_{i}, U_{i+1}\right)=\mathcal{F}\left(U_{i}, U_{i+1}\right)+c_{i+1 / 2}\left(\begin{array}{c}
0 \\
u_{i+1}-u_{i}
\end{array}\right),
$$

and $c_{i+1 / 2}=\max _{a= \pm} \max _{j=i, i+1}\left|\lambda_{a}\left(u_{j}, h_{j}\right)\right|$, with $\lambda_{ \pm}$defined similarly (but in 1D) as in $(6)$.

The reader is referred to $[3,13]$ for generalities on the theory of finite volume method used in hyperbolic systems, and to $[4,12,14]$ for the special case of shallow water applications.

\section{Numerical results}

A major difficulty one encounters when integrating the system (11) or (21) is to find solutions having $h_{i} \rightarrow 0$ for some $i \in I$ and $h_{i} \geq c>0$ for $i \notin I$. In this case, both systems become singular. To fix this problem, we use a semi-implicit method with time-variable discretization. Our full discrete and semi-implicit methods reads as:

$$
\begin{aligned}
h_{i}^{n+1} & =h_{i}^{n}-\lambda\left(\left(h_{i+1 / 2}^{n} \bar{u}_{i+1 / 2}^{n}-h_{i-1 / 2}^{n} \bar{u}_{i-1 / 2}^{n}\right)+\Delta t \mathfrak{M}_{i}^{n},\right. \\
\left(h_{i} u_{i}+\overline{\mathcal{K}}_{i}\left|u_{i}\right| u_{i}\right)^{n+1} & =h_{i}^{n} u_{i}^{n}-\lambda\left[\left(h_{i+1 / 2}^{n+1}\left(\bar{u}_{i+1 / 2}^{n}\right)^{2}-h_{i-1 / 2}^{n+1}\left(\bar{u}_{i-1 / 2}^{n}\right)^{2}\right)-\right. \\
& -\frac{1}{2}\left(h_{i+1 / 2}^{s}\left(w_{i+1}-w_{i}\right)+h_{i-1 / 2}^{s}\left(w_{i}-w_{i-1}\right)\right)^{n+1}+ \\
& \left.+h_{i+1 / 2}^{n+1} c_{i+1 / 2}^{n}\left(u_{i+1}^{n}-u_{i}\right)-h_{i-1 / 2}^{n+1} c_{i-1 / 2}^{n}\left(u_{i}^{n}-u_{i-1}^{n}\right)\right]
\end{aligned}
$$

As our time integration scheme is of semi-implicit type, it is expected that there must be an upper bound for the time step to assure the stability of the numerical scheme. By numerical investigation, we find the following constraint

$$
\lambda<\frac{1}{2 \max _{i} c_{i+1 / 2}} .
$$

for the time step. Note that if the time step satisfies the condition (23) and if there are no mass sources (i.e. $\mathfrak{M}=0$ ) then $h_{i}^{n} \geq 0$ for any $i$ and $n>0$ provided that $h_{i}^{0} \geq 0$. 
Remark 2. The scheme works well for $\mathcal{K}_{i}$ not too small. In our examples, any $\mathcal{K}_{i}>0.1$ is good enough.

Figure 3 presents some numerical results obtained with our scheme.
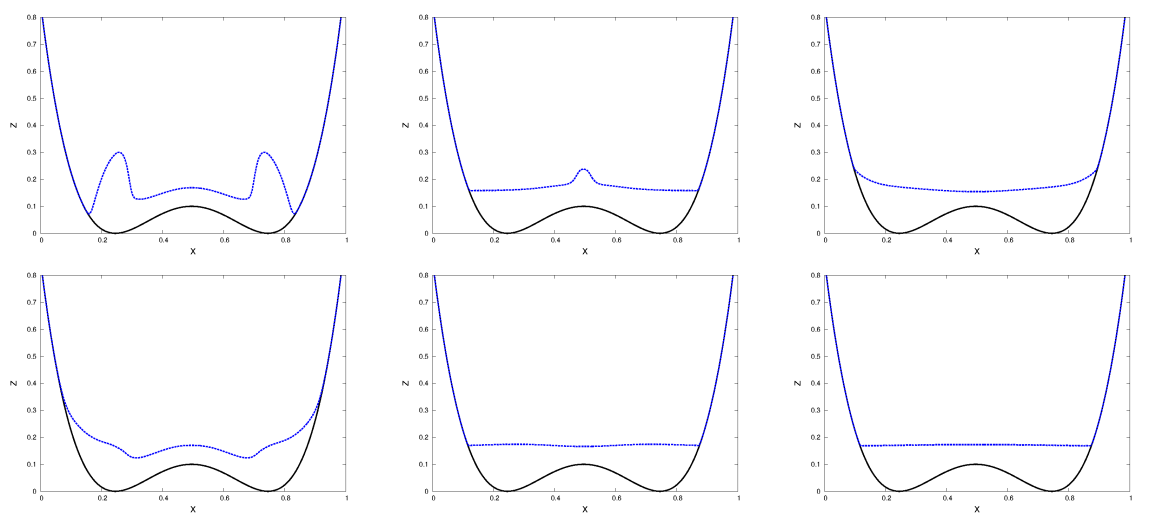

Figure 3: The dashed and continuous lines represent the water and soil surfaces, respectively. The first row of pictures gives the evolution of the free water surface on bare soil, i.e. absence of vegetation, at times $t=0.1,2$, and 3 . The second row gives the evolution of the free water surface on a soil covered with vegetation at the same moments of time as before.

\section{Conclusions and final remarks}

The modelling equations (1) mainly address to a hydrographic basin that exhibits variation with respect to the soil surface orientation, slope and plant cover density. It is quite general and allows one to solve many practical problems. In order to use it, one needs tools for terrain data acquisition concerning physical parameters and some high computational infrastructure. In this paper, we restrict ourselves to a simplified problem and show the flexibility of the model of taking into account the influence of plant cover on the water flow dynamics. We also investigate some mathematical properties (with physical relevance) of the discrete model (11), and the energy dissipation due to water-plant and water-soil surface frictional interactions.

Concerning the time numerical integration of the ODE model, note that the standard integration methods (e.g. Runge-Kutta or Euler) do not work when the thickness of the water film vanishes. Unfortunately, we were not able to find a full discrete variant for the energy dissipation property that helps us to better control the time step. Numerical tests emphasized there exists an 
upper bound of the time step that keeps under control the boundness of the solution. The condition (23) was mainly obtained by analogy with the well known Lax condition used in hyperbolic system theory, and we observed that it also works in our case.

As further research, we plan to improve the time numerical integration of the ODE model and to analyze the convergence of the ODE solution to the solution of the continuum model.

\section{Acknowledgment}

This work was performed within the project 50/2012 ASPABIR (www.aspabir.biogeochemistry.ro) funded by UEFISCDI, Romania.

\section{References}

[1] M.J. Baptist, V. Babovic, J. Rodriguez Uthurburu, M. Keijzer, R.E. Uittenbogaard, A. Mynett and A. Verwey, On inducing equations for vegetation resistance, Journal of Hydraulic Research, 45:4(2007), pp. 435-450.

[2] F. Bouchut and M. Westdickenberg, Gravity driven shallow water models for arbitrary topography, Comm. Math. Sci. 2: 3(2004), pp. 359-389.

[3] F. Bouchut, Nonlinear Stabilty of Finite Volume Methodes for Hyperbolic Conservattion Laws, Birkhauser, 2004.

[4] A. Chinnayya, A.Y. LeRoux, N. Seguin, A well-balanced numerical scheme for the approximation of the shallow-water equations with topography: the resonance phenomenon, International Journal on Finite Volume (electronic), volume 1(1), pp 1-33, 2004.

[5] S.M. Hassanizadeh, W.G. Gray, Mechanics and thermodynamics of multiphase flow in porous media including interphase boundaries, Adv. Water. Resources, 13:4(1990), pp. 169-186.

[6] M.R. Hoffmann, Application of a simple space-time averaged porous media model for flow in densely vegetated channels Journal of Porous Media, 7: 3 (2004), pp. 479-489.

[7] S. Ion, D. Marinescu, S.G. Cruceanu, Overland flow in the presence of vegetation, Technical report, www.ima.ro/PNII_programme/ASPABIR/pub/report_ismma_aspabir_2013.pdf

[8] C.S. James, A.L. Birkhead, A.A. Jordanova, J.J. O'Sullivan, Flow resistance of emergent vegetation, J. of Hydraulic Res., 42:4(2004), 390-398. 
[9] R.J. Lowe, U. Shavit, J.L. Falter, J.R. Koseff, S.G. Monismith, Modeling flow in coral communities with and without waves: A synthesis of porous media and canopy flow approaches, Limnol. Oceanogr., 53:6(2008), pp. $2668-2680$.

[10] G. Dal Maso, P.G. Le Floch, F. Murat, Definition and weak stabilty of noncoservative product, J. Math. Pures Appl. 74 (1995), 483-458.

[11] H.M. Nepf, Drag, turbulance, and diffusion in flow through emergent vegetation, Water Resource Research, 35: 2(1999), pp. 479-489. Flow in Porous Media I: A Theoretical Derivation of Darcy's Law, Transport in Porous Media 1 (1986), 3-25.

[12] S. Noelle, N. Pankratz, G. Puppo, J.R. Natvig, Well-balanced finite volume schemes of arbitrary order of accuracy for shallow water flows, Journal of Computational Physics, 213 (2006), pp. 474-499.

[13] R.L. LeVeque, Finite Volume Methods foe Hyperbolic Problems, Cambridge University Press, 2002.

[14] U.S. Fjordholm, S. Mishra, E. Tadmor, Well-balanced and energy stable schemes for the shallow water equations with discontinuous topography, Journal of Computational Physics, 230 (2011), pp. 5587-5609.

[15] W. Wu, F.D. Shields Jr., S.J. Bennett, S.S.Y. Wang, A depth-averaged two-dimensional model for flow, sediment transport, and bed topography in curved channels with riparian vegetation, 41, 3(2005), pp. 1-15.

Stelian ION, Email: stelian.ion@ima.ro

Dorin MARINESCU, Email: dorin.marinescu@ima.ro

Anca Veronica ION, Email: anca-veronica.ion@ima.ro

Stefan Gicu CRUCEANU, Email: stefan.cruceanu@ima.ro

Institute of Statistical Mathematics and Applied Mathematics

Romanian Academy

Calea 13 Septembrie, No. 13, 050711 Bucharest, Romania.

Virgil IORDACHE

Research Center for Ecological Services

University of Bucharest

Splaiul Independentei, No. 91-95, 050089 Bucharest, Romania.

Email: virgil.iordache@bio.unibuc.ro 